\title{
PREVALENCE AND ANTIBIOTIC RESISTANCE OF ENTEROCOCCUS STRAINS ISOLATED FROM POULTRY
}

\author{
Dagmara StęPIEŃ-PYŚniAK ${ }^{*}$, Agnieszka MAREK ${ }^{1}$, Tomasz BANACH ${ }^{2}$, \\ Łukasz ADASZEK ${ }^{2}$, Ewelina PYZIK ${ }^{1}$, Jarosław WILCZYŃSKI ${ }^{3}$ and Stanisław WINIARCZYK ${ }^{2}$ \\ ${ }^{1}$ Sub-Department of Veterinary Prevention and Avian Diseases, Institute of Biological \\ Bases of Animal Diseases and ${ }^{2}$ Department of Epizootiology and Clinic of Infectious \\ Diseases, Faculty of Veterinary Medicine, University of Life Sciences in Lublin, \\ Głęboka 30, 20-612 Lublin, Poland; ${ }^{3}$ Veterinary Diagnostic Laboratory Lab-Vet, \\ Tarnowo Podgórne, Poland
}

(Received 2 June 2015; accepted 4 May, 2016)

The aim of this study was to evaluate the frequency of occurrence of bacteria of the genus Enterococcus in poultry, to identify them by means of matrixassisted laser desorption/ionisation time-of-flight mass spectrometry (MALDITOF MS), and to analyse the antimicrobial susceptibility of the isolated strains to the drugs most frequently used in poultry. The material for the bacteriological tests was obtained mainly from the heart $(97 \%)$ of the birds investigated. Of a total of 2,970 samples tested, 911 (30.7\%) tested positive for Enterococcus spp. Enterococci were detected in broilers $(88.1 \%)$, laying hens $(5.3 \%)$, turkeys $(3.9 \%)$, breeding hens $(2.2 \%)$, and geese $(0.4 \%)$. The most commonly identified species were Enterococcus (E.) faecalis $(74.7 \%)$, E. faecium (10.1\%), E. gallinarum $(5.5 \%)$, E. hirae $(4.6 \%)$, and E. cecorum $(4.1 \%)$. The most frequent resistance properties were resistance to sulphamethoxazole/trimethoprim $(88 \%)$, tylosin (71.4\%), enrofloxacin (69.4\%), doxycycline (67.3\%), and lincomycin/spectinomycin (56.1\%). Only one vancomycin-resistant Enterococcus, E. cecorum from a broiler, was found.

Key words: Enterococcus, poultry, antibiotic resistance, MALDI-TOF MS

Enterococci are part of the normal intestinal flora of animals and man. Despite their small share in the microbiota of the macroorganism, an increase in the clinical significance of these opportunistic pathogens is being observed. Enterococcus cecorum is mainly associated with arthritis, spondylitis, osteomyelitis, spondylolisthesis, and femoral head necrosis in broiler and broiler breeder flocks (Devriese et al., 2002; De Herdt et al., 2008; Stalker et al., 2010; Makrai et al., 2011; Szeleszczuk et al., 2013). Enterococcus faecalis has been linked to endocarditis in chickens, hepatic granulomas in turkeys, ascites in hens, pulmonary hypertension in broilers (Tankson et al., 2001), and amyloid arthropathy with

*Corresponding author; E-mail: dagmara.stepien@up.lublin.pl 
systemic amyloidosis in broiler breeders (Steentjes et al., 2002). Enterococcus durans has been found in young chickens with bacteraemia and encephalomalacia (Abe et al., 2006), while E. hirae has been reported in cases of focal necrosis of the brain in young chicks and in broilers with osteomyelitis and endocarditis (Kolbjørnsen et al., 2011; Velkers et al., 2011).

Species identification of unusual enterococci by routine standard methods is not always reliable, particularly in the case of strains of veterinary importance. Moreover, the occurrence of atypical phenotypic characteristics in some microorganisms may also lead to misidentification (Tsakris et al., 1998). Correct identification of enterococci is crucial for epidemiological and therapeutic purposes. A relatively new identification method using a MALDI-TOF MS system with prior formic acid extraction has provided excellent diagnostic results and reduced identification time (Seng et al., 2009; Wieser et al., 2012). MALDI-TOF MS is a rapid and accurate technique for the identification of various types of Grampositive or Gram-negative bacteria (Kosikowska et al., 2014; Marek et al., 2015; Nowakiewicz et al., 2015).

Over the years, bacterial pathogens have developed resistance to various antibiotics. The main risk factor for increased antibiotic resistance is the extensive use of antibiotics in agriculture, which leads to the emergence and dissemination of resistant bacteria and resistance genes in animals (Aarestrup et al., 2008).

The aim of this study was to evaluate the frequency of occurrence of bacteria of the genus Enterococcus in poultry, identify them by MALDI-TOF mass spectrometry (Bruker Daltonics, Germany) and test them for susceptibility to the drugs most frequently used in poultry, as well as to vancomycin. In addition, antimicrobial resistance of E. faecalis and E. faecium strains isolated from broilers, layers and turkeys was evaluated using the Minimum Inhibitory Concentration (MIC) technique with vancomycin, ampicillin, gentamicin, and erythromycin.

\section{Materials and methods}

\section{Material}

Between October 2013 and September 2014, a total of 2,970 tests were performed. The samples were collected from 580 poultry flocks, including 420 broiler flocks, 80 turkey flocks, 73 laying and breeding hen flocks, and 7 geese flocks.

The material for the bacteriological tests consisted mainly of hearts (97\%), as well as of livers, brains, bone marrow, and oviduct swabs (3\%), from poultry of different species and production purposes, aged from 1 day to 60 weeks. The birds examined were most commonly of less than 10 days of age. Samples were most frequently taken from broilers, layers, turkeys and geese. Pathological findings in the affected birds were increased mortality, poorer weight gain, decreased 
laying capacity in hens, salpingitis, yolk sac infection, arthritis, bone marrow infections, spondylitis, femoral head necrosis, and endocarditis.

\section{Bacteriological analysis}

Bacteria of the genus Enterococcus were isolated on the differentialselective medium Bile Esculin Azide Lab-Agar (BIOCORP, Poland) and Blood Lab Agar (BIOCORP, Poland) supplemented with 5\% defibrinated horse blood, at $37^{\circ} \mathrm{C}$ for $24-48 \mathrm{~h}$ under microaerophilic conditions. The bacterial isolates were initially characterised based on their colony morphology, Gram stain morphology, the presence and type of haemolysis, production of catalase, and activity of pyrrolidonyl arylamidase (PYRAtest, Erba Lachema, Czech Republic).

\section{Identification of isolated strains by MALDI-TOF MS analysis}

The isolated bacteria were identified using MALDI-TOF mass spectrometry (Bruker Daltonics, Germany). The identification step was preceded by a preliminary extraction of proteins with ethanol and formic acid. For this purpose, a single colony of a fresh 18- to 24-h culture grown on Blood Lab Agar (BIOCORP, Poland) supplemented with $5 \%$ defibrinated horse blood at $37^{\circ} \mathrm{C}$ was suspended in $300 \mu \mathrm{l}$ of sterile deionised water, after which $900 \mathrm{ml}$ of pure ethanol (POCH) was added and the sample was mixed thoroughly by vortexing. The sample was then centrifuged for $2 \mathrm{~min}$ at 13,000 rpm. After the supernatant was discarded, $50 \mathrm{ml}$ of $70 \%$ aqueous formic acid and then $50 \mathrm{ml}$ of acetonitrile (Fluka Analytical) were added to the precipitate, and the sample was thoroughly mixed by vortexing. After centrifugation (13,000 rpm for $2 \mathrm{~min}), 1 \mu \mathrm{l}$ of the supernatant was collected, applied to a metal plate and allowed to dry at room temperature. Then $1 \mu \mathrm{l}$ of HCCA ( $\alpha$-Cyano-4-hydroxycinnamic acid) matrix solution was applied to each dosed bacterial sample and left to dry at room temperature. Automatic measurement of the spectrum and comparative analysis with reference spectra of bacteria was performed using an Ultraflextreme mass spectrometer and MALDIBiotyper 3.0 software (Bruker Daltonics, Germany). The analysis was repeated three times for each sample. The reliability of identification in the MALDI Biotyper system was expressed in points. Scores $\geq 2.0$ indicated identification to the species level. Six enterococcal strains obtained from the American Type Culture Collection (ATCC) were used as control. These strains represent the following species: E. faecalis (ATCC29212, ATCC19433), E. faecium (ATCC19434), E. casseliflavus (ATCC49996), E. gallinarum (ATCC49573), and E. hirae (ATCC8043).

\section{Dendrogram construction for $\mathrm{E}$. cecorum}

Due to the large number of isolated enterococci and the growing interest in E. cecorum infection associated with its typical clinical symptoms in broiler and 
broiler breeder flocks worldwide, we created a dendrogram for this species only. Based on cross-wise minimum spanning tree (MSP) matching, the dendrogram was created with similar MSPs, resulting in a high matching score value. Each MSP was matched against all MSPs of the analysed set. The list of score values was used to calculate normalised distance values between strains, resulting in a matrix of matching scores. The visualisation of the respective relationship between the MSPs was displayed in a dendrogram using MALDI Biotyper 3.0 software (Bruker Daltonik, Germany) (Sauer et al., 2008).

\section{Susceptibility testing}

The sensitivity of the isolated strains to selected antibiotics and chemotherapeutics commonly used to treat poultry was tested using the Kirby-Bauer disk diffusion method on Mueller-Hinton agar (BioMérieux, France) (CLSI, 2008). The results were read and interpreted based on the diameter of the inhibition zone, with the strains designated as resistant (R), of intermediate sensitivity (I) or sensitive (S). The sensitivity profiles of the bacteria were determined for the following agents (OXOID, Hampshire, UK): vancomycin (VA $30 \mu \mathrm{g}$ ), amoxicillin (AML 25 $\mu \mathrm{g}$ ), amoxicillin with clavulanic acid (AMC $30 \mu \mathrm{g}$ ), doxycycline (DO $30 \mu \mathrm{g}$ ), enrofloxacin (ENR $5 \mu \mathrm{g}$ ), florfenicol (FFC $30 \mu \mathrm{g}$ ), lincomycin/spectinomycin (LS $109 \mu \mathrm{g}$ ), tylosin (TY $30 \mu \mathrm{g}$ ), and sulphamethoxazole/trimethoprim (SXT $25 \mu \mathrm{g}$ ).

In addition, for compelling reasons of public health, further tests were carried out to determine sensitivity to vancomycin $(0.125-64 \mu \mathrm{g} / \mathrm{ml})$, ampicillin $(0.125-$ $64 \mu \mathrm{g} / \mathrm{ml})$, gentamicin $(2-1024 \mu \mathrm{g} / \mathrm{ml})$ and erythromycin $(0.125-64 \mu \mathrm{g} / \mathrm{ml})$ using the Minimal Inhibitory Concentration method. As the largest proportion of tested samples came from broilers and therefore a very large number of bacterial strains were isolated from these birds, for the analysis of antibiotic resistance we selected E. faecalis and E. faecium strains from broilers only in cases where the highest mortality rates were noted in the flock (40-60 to 100-120 per day) or where the pathological signs indicated bacterial infection. The MICs of antimicrobial agents representing four classes were determined in accordance with the guidelines of the Clinical and Laboratory Standards Institute (CLSI, 2008).

The results of the drug sensitivity tests for the E. faecalis (ATCC29212) reference strain were used as the reference system.

\section{Results}

\section{Bacteriological analysis and identification by MALDI-TOF MS}

A total of 911 samples (30.7\%) were positive for Enterococcus spp. The MALDI-Biotyper 3.0 successfully identified all isolates to species. The mean identification log score for all tested strains was 2.293. Enterococci were detected 
in broilers $(88.1 \%)$, laying hens $(5.3 \%)$, turkeys $(3.9 \%)$, breeding hens $(2.2 \%)$ and geese $(0.4 \%)$. Bacteria of the genus Enterococcus were most frequently isolated from birds at the age of $1-3$ days $(87.3 \%)$, but also at the age of 4-10 days (3.2\%), 2-4 weeks (5.5\%), 5-7 weeks (1.8\%), and 12-60 weeks (2.2\%). A wide variety of Enterococcus species were distinguished among the isolates. The most predominant species were identified as E. faecalis $(74.7 \%)$, E. faecium $(10.1 \%)$, E. gallinarum (5.5\%), E. hirae (4.6\%), and E. cecorum (4.1\%). The remaining strains were E. casseliflavus $(0.8 \%), E$. avium $(0.1 \%)$ in the heart of a 23 -weekold laying hen, and E. columbae $(0.1 \%)$ in a 2 -week-old goose. The bacteria of the genus Enterococcus isolated from different species of poultry with different production purposes are presented in Table 1.

Table 1

The number and relative frequencies of isolates of Enterococcus species in different groups of poultry

\begin{tabular}{lcccccc}
\hline $\begin{array}{l}\text { Enterococcus } \\
\text { species }\end{array}$ & $\begin{array}{c}\text { Breeding hens } \\
(\mathrm{n}=20)\end{array}$ & $\begin{array}{c}\text { Laying hens } \\
(\mathrm{n}=48)\end{array}$ & $\begin{array}{c}\text { Broilers } \\
(\mathrm{n}=803)\end{array}$ & $\begin{array}{c}\text { Turkeys } \\
(\mathrm{n}=36)\end{array}$ & $\begin{array}{c}\text { Geese } \\
(\mathrm{n}=4)\end{array}$ & $\begin{array}{c}\text { Total } \\
(\mathrm{n}=911)\end{array}$ \\
\hline E. cecorum & $3(15 \%)$ & $6(12.5 \%)$ & $27(3.4 \%)$ & 0 & $1(25 \%)$ & $37(4.1 \%)$ \\
E. faecalis & $12(60 \%)$ & $34(70.8 \%)$ & $611(76.1 \%)$ & $22(61.1 \%)$ & $2(50 \%)$ & $681(74.7 \%)$ \\
E. faecium & $2(10 \%)$ & $2(4.2 \%)$ & $86(10.7 \%)$ & $2(5.6 \%)$ & 0 & $92(10.1 \%)$ \\
E. hirae & 0 & $4(8.3 \%)$ & $36(4.5 \%)$ & $2(5.6 \%)$ & 0 & $42(4.6 \%)$ \\
E. gallinarum & $3(15 \%)$ & $1(2.1 \%)$ & $36(4.5 \%)$ & $10(27.7 \%)$ & 0 & $50(5.5 \%)$ \\
E. casseliflavus & 0 & 0 & $7(0.8 \%)$ & 0 & 0 & $7(0.8 \%)$ \\
E. avium & 0 & $1(2.1 \%)$ & 0 & 0 & 0 & $1(0.1 \%)$ \\
E. columbae & 0 & 0 & 0 & 0 & $1(25 \%)$ & $1(0.1 \%)$ \\
\hline
\end{tabular}

\section{Dendrogram for E. cecorum}

The dendrogram for E. cecorum is shown in Fig. 1. Dendrogram analysis indicates that E. cecorum isolated from broiler chickens (BC), laying hens (LH), breeding hens $(\mathrm{BH})$ and geese $(\mathrm{G})$ have a similar protein profile, regardless of the species of birds from which they were isolated. The bacterial isolates obtained can be classified into two main phylogenetic groups: one (cluster 1) contained only 4 isolates of E. cecorum ( 3 isolated from BC and one from $\mathrm{LH}$ ), while the other strains $(n=33)$ obtained in our study, together with the reference strains $(\mathrm{n}=2)$, were in the second phylogenetic group (cluster 2$)$.

\section{Susceptibility testing}

Resistance to two or more antibiotic agents was demonstrated in all of the isolated enterococci. High resistance $(>50 \%$ resistant) to sulphamethoxazole/ trimethoprim [SXTg (88\%), tylosin (71.4\%), enrofloxacin (69.4\%), doxycycline $(67.3 \%)$, and lincomycin/spectinomycin $(56.1 \%)$ was shown in all isolates. 
Moreover, a certain percentage of isolates exhibited intermediate sensitivity, particularly to enrofloxacin (6.7\%), lincomycin/spectinomycin (4.5\%), florfenicol $(3.3 \%)$, doxycycline $(3 \%)$, and tylosin $(2.3 \%)$.

Resistance to vancomycin $(0.11 \%)$, amoxicillin (4\%), amoxicillin with clavulanic acid (4.5\%) and florfenicol (15.7\%) was classified as low $(<25 \%)$. However, none of the E. cecorum, E. casseliflavus, E. avium or E. columbae strains was found to be resistant to amoxicillin and amoxicillin with clavulanic acid. Detailed data are presented in Table 2.

Resistance of E. faecalis and E. faecium isolates to selected antimicrobial agents recommended by the CLSI is shown in Table 3. Due to the limited numbers of E. faecium isolates from layers and turkeys available for susceptibility testing, only isolates of E. faecalis were analysed in detail to compare the results between different poultry species.

The majority of $E$. faecalis isolates were resistant or intermediate-resistant to gentamicin $(91.7 \%, 82.4 \%$ and $68.2 \%)$ and erythromycin $(52.8 \%, 88.2 \%$ and $100 \%)$ in broilers, layers and turkeys, respectively. The highest resistance to gentamicin was obtained in E. faecalis isolates from broilers (51.4\%). Furthermore, high-level aminoglycoside (gentamicin) resistance was noted in two E. faecalis strains isolated from broilers $(1,024 \mu \mathrm{g} / \mathrm{ml})$. The percentage of strains resistant to erythromycin varied from 33.4 and 70.6 for broilers and layers, respectively, to 100 for turkeys.

Vancomycin-resistant isolates could not be detected in either Enterococcus species. However, $1.4 \%$ of E. faecalis and $10.5 \%$ of E. faecium strains isolated from broilers were intermediate-resistant $(8 \mu \mathrm{g} / \mathrm{ml})$ to vancomycin. All of the tested E. faecalis and E. faecium isolates showed sensitivity to ampicillin.

\section{Discussion}

Enterococcus faecium and E. faecalis are usually the most prevalent enterococcal species among isolates recovered from environmental samples such as poultry faeces/manure, feed, water and air (Yoshimura et al., 2000; Ruzauskas et al., 2009; Ali et al., 2013; Furtula et al., 2013), but very few data are available on enterococci from internal organs of healthy or diseased poultry (Tankson et al., 2002; Maasjost et al., 2015). In contrast to these data, the results obtained in this study indicate that E. faecalis accounted for the highest percentage of enterococci isolated from the internal organs of poultry, followed by E. faecium. In studies carried out in other countries, E. faecalis was also the predominant Enterococcus species in faecal samples from poultry (Yoshimura et al., 2000; Kuhn et al., 2003; Poeta et al., 2006). According to the literature, chickens are initially colonised by E. faecalis (Fertner et al., 2011), but this population is then displaced, mainly by E. faecium (Kaukas et al., 1987). In this study, the dominance 
of $E$. faecalis could be linked to the age of the birds tested. Most of the birds examined were at the age of 1 to 3 days $(87.3 \%)$.

Like in this our study, a low prevalence of E. gallinarum, E. casseliflavus, $E$. hirae, E. durans and E. mundtii was found in the reports cited above. Nowakiewicz et al. (2014) noted that, besides poultry, a small number of $E$. hirae, E. durans and E. mundtii can also be isolated from the gastrointestinal tract of foxes (Vulpes vulpes), more frequently than from other domestic animals tested. Some enterococci are considered to be associated with a particular host species. Enterococcus columbae is thought to be specific to pigeons (Devriese et al., 1990), but we isolated this strain from a 2-week-old goose raised in intensive poultry production. Enterococcus columbae does not grow on selective media commonly used for the isolation of enterococci and it requires $\mathrm{CO}_{2}$ for growth, so the prevalence of this species may be underestimated. It should be noted that E. gallinarum, originally described in chickens, was not found in faecal samples from poultry of unspecified age by Tejedor-Junco et al. (2005). In our study on heart samples, E. gallinarum was the third most frequently isolated species in young ( $<10$ days old) broilers. More importantly, it was the second most frequent Enterococcus species in turkeys. The results obtained by Tankson et al. (2002) suggested that the heart and lungs of healthy young chickens do not have a residual bacterial flora, but rather have a wide variety of opportunistic bacteria occasionally passing through these tissues in the post-hatching period. However, it is possible that some of these bacteria - like enterococci - could produce pathologic lesions if predisposing conditions prevail.

Enterococcus cecorum was found particularly frequently in breeders and layers. Age-dependent colonisation by E. faecalis and E. cecorum was confirmed in this study. The composition of the commensal flora of the poultry intestine may change under the influence of diet, age, stress, type of litter, and especially antibiotic use (Burkholder et al., 2008; Torok et al., 2009, 2011). According to the literature, E. cecorum infection appears to be most common in broiler flocks at the age of 3-6 weeks, and in broiler breeders aged 3.5-18 weeks. (Devriese et al., 2002; De Herdt et al., 2008; Armour et al., 2011; Makrai et al., 2011). In our study, the highest percentage of E. cecorum was isolated from broilers at the age of $2-5$ weeks. In addition, we identified these bacteria in laying hens (2235 weeks), breeding hens (28-37 weeks), and geese (50 days).

MALDI-TOF MS and the Biotyper software allows the reliable species assignment of difficult but important pathogens such as E. cecorum. MALDI Biotyper also demonstrated a similar protein profile of E. cecorum isolated from different kinds of poultry. The mass spectra dendrogram provides information not only about correlations between MSPs but also about possible changes in bacterial strains with respect to the standard strain. The changes in protein profile are clearly and strongly correlated with a rapid response of bacterial strains to environmental changes. 


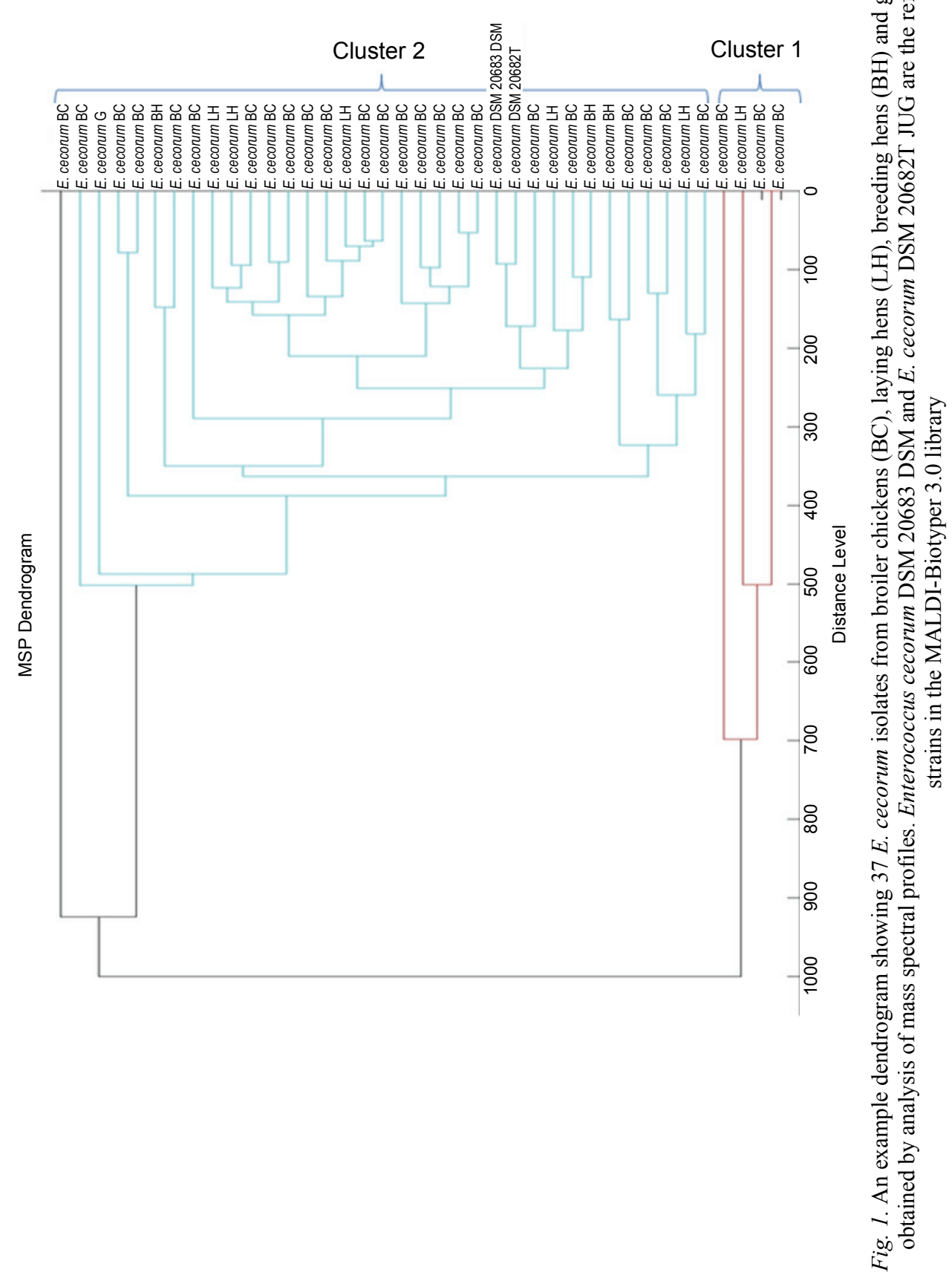




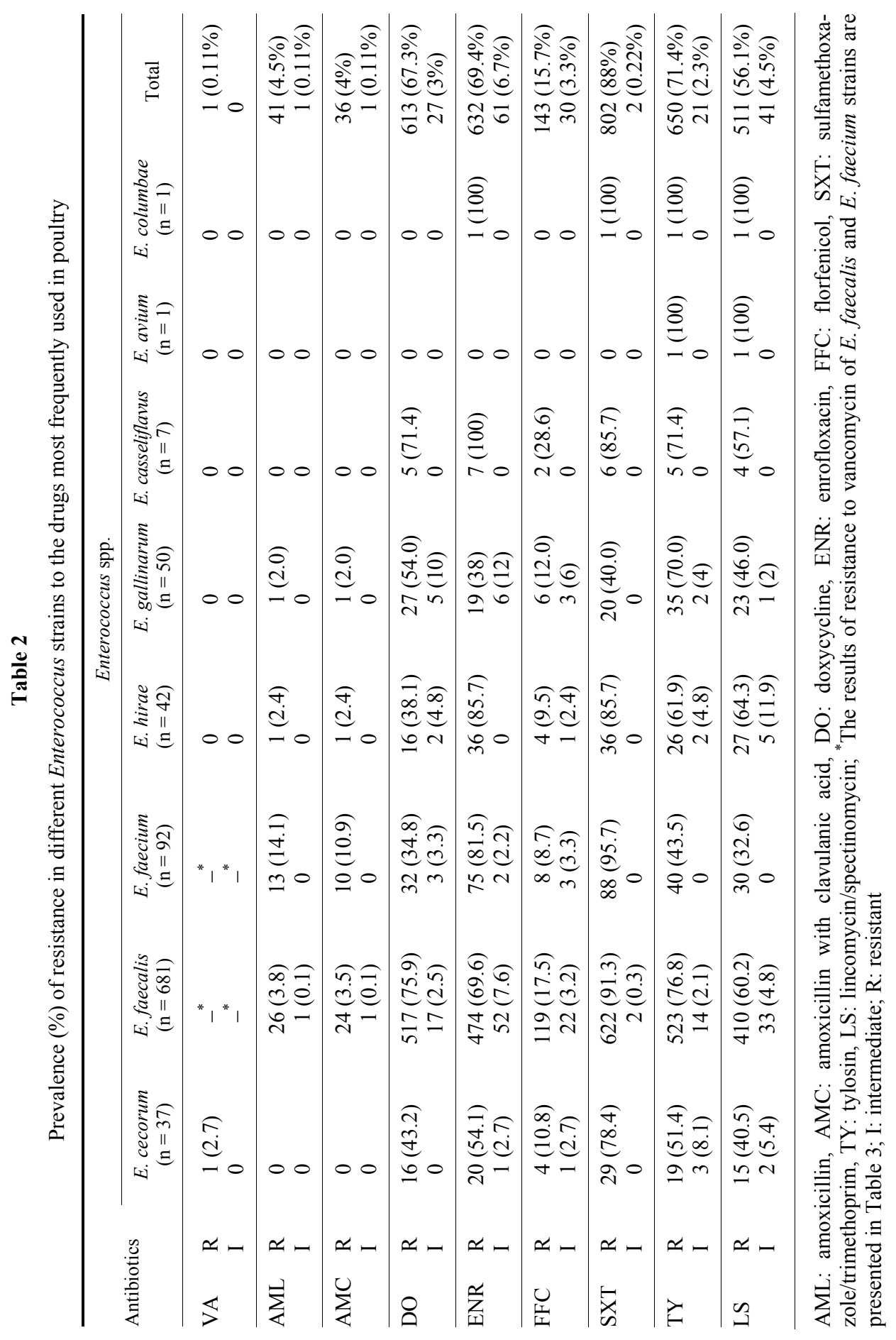




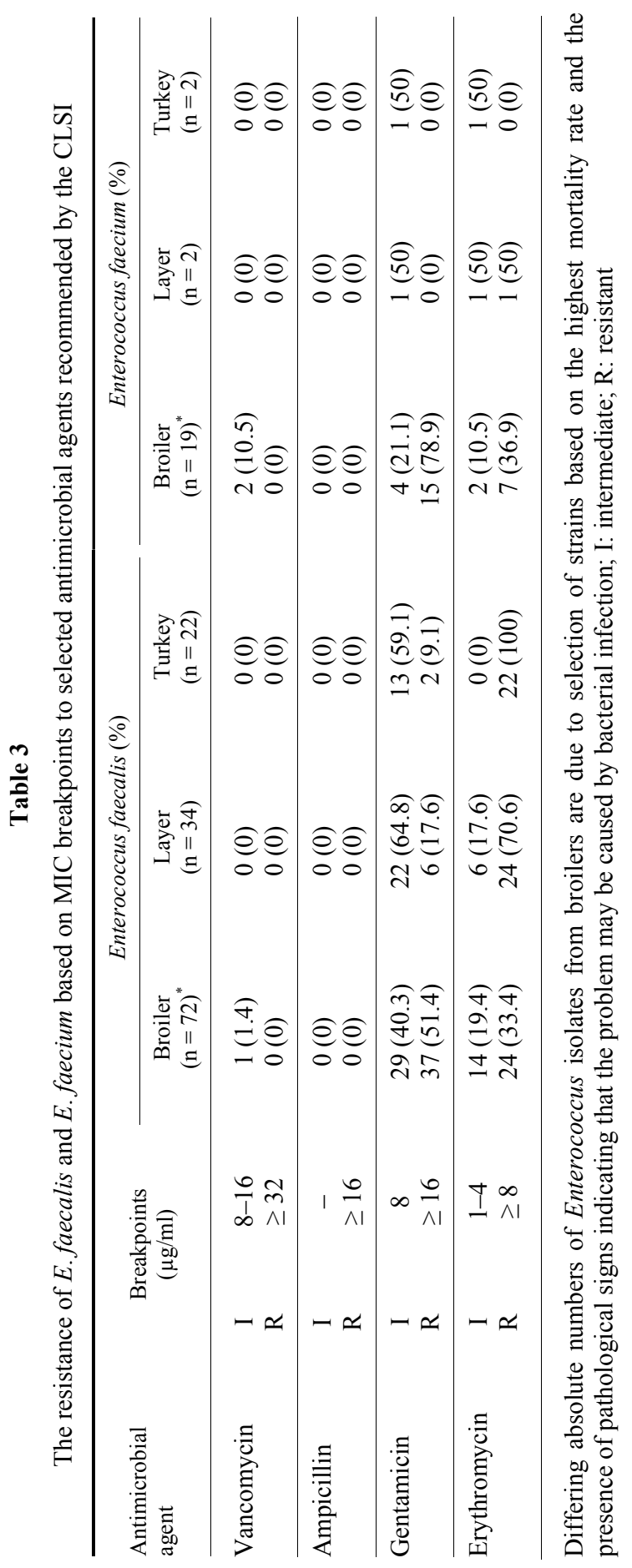


The antibiotic sensitivity tests showed that all of the strains were resistant to more than one of the agents applied. The highest level of resistance was detected for sulphamethoxazole/trimethoprim. Makrai et al. (2011) also observed that all tested strains were resistant to SXT. Bacteria of the genus Enterococcus can absorb folic acid from the environment, bypassing the effects of SXT. Therefore, in vitro testing of enterococcal susceptibility to these agents in a medium devoid of folate would yield a more meaningful result.

In the present study, we noted a high frequency of resistance to tylosin, enrofloxacin, doxycycline, and lincomycin/spectinomycin. Our results are similar to the resistance profiles of lincomycin, tetracycline, penicillin, ciprofloxacin and tylosin in Enterococcus isolates from the environment of broiler and/or layer farms (Ruzauskas et al., 2009; Diarra et al., 2010; Šeputienè et al., 2012; Furtula et al., 2013). Moreover, in Poland, Różańska et al. (2015) noted that the highest number of $E$. faecalis strains isolated from poultry meat were resistant to lincomycin (24 strains; 100\%), the second-highest resistance was to tetracycline (21 strains; $87.5 \%$ ), followed by tylosin (16 strains; 66.7\%). Erythromycin resistance was also notable (7 strains; $29.2 \%$ ). In our study, erythromycin resistance of $E$. faecalis isolates ranged from $33.4 \%(\mathrm{n}=72)$ in broilers and $70.6 \%(\mathrm{n}=24)$ in layers to $100 \%(\mathrm{n}=22)$ in turkeys.

After the European Union banned the use of growth promoters in animal production, there were some increases in morbidity and mortality among farm animals, which entailed a proportional increase in the therapeutic and preventive administration of antibiotics (Cogliani et al., 2011). The use of antimicrobials inevitably leads to the selection of resistant bacterial strains in the ecosystem. Enterococcus isolates having an $\mathrm{MLS}_{\mathrm{B}}$ (macrolide-lincosamide-streptogramin B) phenotype confer high-level resistance not only to macrolides (tylosin, erythromycin) but also to lincosamides. Enterococci, which express the erm(B) gene, often exhibit resistance to tetracycline in addition to resistance to $\mathrm{MLS}_{\mathrm{B}}$. Cauwerts et al. (2007) observed that in $89 \%$ of erm(B)-positive enterococci, tet genes were also present. Therefore, the frequent use of tetracyclines in poultry may co-select for resistance to $\mathrm{MLS}_{\mathrm{B}}$ antibiotics, which may be important as an alternative therapy for enterococcal infections in humans.

Kuo et al. (2009) showed that point mutations in the genome of E. faecalis can be used to generate resistance to quinolones in healthy chickens and pigs. The incidence of such mutations depends on the intensity of antibiotic therapy with fluoroquinolones. Thus, high resistance to enrofloxacin can be associated with the administration of this drug at a therapeutic level for short periods of time or for preventive use in growing broilers. Resistance to fluoroquinolones was frequently observed in E. faecium (82.8\%) and E. faecalis $(17.9 \%)$ in countries of Southeast Asia (Usui et al., 2014). According to Maasjost et al. (2015), fewer isolates, numbering 7 (5\%) of E. faecalis and 10 (56\%) of E. faecium from the internal organs of poultry, demonstrated resistance to ciprofloxacin. In the 
present study, resistance to enrofloxacin was observed at a higher frequency among E. faecium (81.5\%), E. hirae (85.7\%) and E. faecalis (69.6\%) isolates. According to Różańska et al. (2015), only a few strains from poultry meat were resistant to ciprofloxacin $(8.3 \%)$ in Poland.

In contrast to Diarra et al. (2010) and Furtula et al. (2013), we found a high sensitivity to penicillin antibiotics in 911 of the isolated Enterococcus strains. Moreover, E. faecalis and E. faecium strains isolated from affected broilers, layers and turkeys were susceptible to ampicillin. Similarly, Różańska et al. (2015) noted that all 24 E. faecalis strains tested from poultry meat were susceptible to penicillin. According to the literature, ampicillin/amoxicillin and macrolides are the antibiotics of choice for E. cecorum infections in poultry (Devriese et al., 2002; De Herdt et al., 2008). We noted that all isolates of this species were susceptible to amoxicillin and amoxicillin with clavulanic acid, while $50 \%$ of them were resistant to tylosin. Makrai et al. (2011) observed that all of the isolated E. cecorum strains were susceptible to amoxicillin and florfenicol, but about half of the isolates were resistant to lincomycin and spectinomycin. The mechanism of resistance to chloramphenicol in enterococci is enzymatic inactivation by acetylation of the drug via different types of chloramphenicol acetyltransferase (Cats). Cats are able to inactivate chloramphenicol as well as thiamphenicol. Florfenicol, however, due to its structural modification (the hydroxyl group $-\mathrm{OH}$ of thiamphenicol is replaced with fluorine $-\mathrm{F}$ ), is resistant to inactivation by these enzymes. This is why we observed low resistance to florfenicol in E. faecium (8.6\%), E. hire (9.5\%), E. cecorum (10.8\%), E. gallinarum $(12 \%)$ and E. faecalis (17.5\%). Higher resistance to chloramphenicol in E. faecium $(80.8 \%)$ and E. faecalis $(21.2 \%)$ isolated from chicken faeces was observed by Ali et al. (2013).

In our study, we did not detect resistance to vancomycin in the E. faecalis and E. faecium isolates but we found one vancomycin-resistant Enterococcus (VRE) - E. cecorum - among 37 such strains isolated from broiler chickens. We also noted intermediate susceptibility of E. faecium and E. faecalis from broilers in $10.5 \%$ and $1.4 \%$ of strains, respectively. Similarly, Maasjost et al. (2015) showed the absence of VRE from the internal organs of affected broilers, layers and turkeys. However, Sting et al. (2013) detected VRE in cloacal and dust samples from 20 turkey flocks in south-western Germany. The European Union summary report of 2013 indicated an overall low-level resistance to vancomycin in E. faecium $(0.1 \%)$ and E. faecalis $(0.6 \%)$ isolated from broiler flocks (EFSA, 2015).

Therefore, differences in the degree of resistance to commonly used antibiotics in poultry may reflect differences in the use of drugs in animal production practices in specific geographic regions (EMA, 2014; EFSA, 2015).

The present study provides original data on the prevalence and antimicrobial resistance of bacteria of the genus Enterococcus isolated from poultry in Poland. The most common species of enterococci from the internal organs of af- 
fected birds were identified as E. faecalis and E. faecium, followed by E. gallinarum, E. hirae and E. cecorum. We observed a few typical clinical signs of $E$. cecorum and E. hirae infections. We found spondylitis, arthritis, bone marrow infection (E. cecorum) and endocarditis (E. hirae). Unfortunately, most of the Enterococcus species caused infections together with other bacteria, so that conclusions could not be drawn about their pathogenic significance. It is worth noting that a surprisingly high percentage of strains were resistant to several of the antibiotics most frequently used in poultry. The presence of a significant percentage of strains of intermediate sensitivity is also a cause for concern.

Vancomycin-resistant E. cecorum and intermediate susceptibility to vancomycin in E. faecalis and E. faecium strains were found only in broilers. Two $E$. faecalis isolates from broiler chickens showed a high level of resistance to gentamicin as well. Interestingly, a small percentage of E. faecalis and E. faecium strains isolated from layers and turkeys were resistant to low levels of gentamicin. Moreover, all of the tested enterococci from layers and turkeys were susceptible to vancomycin and ampicillin. Moreover, we observed a high frequency of sensitivity to amoxicillin, amoxicillin with clavulanic acid and florfenicol.

In conclusion, this study confirms that the use of antibiotics and chemotherapeutic compounds, especially in intensive poultry production, has led to expansion of the population of multiresistant Enterococcus strains in the poultry population of Poland, while vancomycin-resistant enterococci $(0.11 \%$ of all isolates) have remained at a low level.

\section{References}

Aarestrup, F. M., Wegener, C. H. and Collignon, P. (2008): Resistance in bacteria of the food chain: epidemiology and control strategies. Expert. Rev. Anti. Infect. Ther. 6, 733-750.

Abe, Y., Nakamura, K., Yamada, M. and Yamamoto, Y. (2006): Encephalomalacia with Enterococcus durans infection in the brain stem and cerebral hemisphere in chicks in Japan. Avian Dis. 50, 139-141.

Ali, S. A., Hasan, K. A., Bin Asif, H. and Abbasi, A. (2013): Environmental enterococci: I. Prevalence of virulence, antibiotic resistance and species distribution in poultry and its related environment in Karachi, Pakistan. Lett. Appl. Microbiol. 58, 423-432.

Armour, N. K., Collett, S. R. and Williams, S. M. (2011): Enterococcus cecorum-related arthritis and osteomyelitis in broilers and broiler breeders. Poult. Inf. Profess. 17, 1-7.

Burkholder, K. M., Thompson, K. L., Einstein, M. E., Applegate, T. J. and Patterson, J. A. (2008): Influence of stressors on normal intestinal microbiota, intestinal morphology, and susceptibility to Salmonella enteritidis colonization in broilers. Poult. Sci. 87, 1734-1741.

Cauwerts, K., Decostere, A., De Graef, E. M., Haesebrouck, F. and Pasmans, F. (2007): High prevalence of tetracycline resistance in Enterococcus isolates from broilers carrying the erm(B) gene. Avian Pathol. 36, 395-399.

CLSI (2008): Document M31-A3. Performance Standards for Antimicrobial Disk and Dilution Susceptibility Tests for Bacteria Isolated from Animals; Approved Standard. 3rd edition. Clinical and Laboratory Standards Institute, Wayne, PA. 
Cogliani, C., Goosens, H. and Greco, C. (2011): Restricting antimicrobial use in food animals: lessons from Europe. Microbe 6, 274-279.

De Herdt, P., Defoort, P., van Steelant, J., Swam, H., Tanghe, L., van Goethem, S. and Vanrobaeys, M. (2008): Enterococcus cecorum osteomyelitis and arthritis in broiler chickens. Vlaams Diergen. Tijds. 78, 44-48.

Devriese, L. A., Cauwerts, K., Hermans, K. and Wood, A. M. (2002): Enterococcus cecorum septicemia as a cause of bone and joint lesions resulting in lameness in broiler chickens. Vlaams Diergen. Tijds. 71, 219-221.

Devriese, L. A., Ceyssens, K., Rodrigues, U. M. and Collins, M. D. (1990): Enterococcus columbae, a species from pigeon intestines. FEMS Microbiol. Lett. 59, 247-251.

Diarra, M. S., Rempel, H., Champagne, J., Masson, L., Pritchard, J. and Topp, E. (2010): Distribution of antimicrobial resistance and virulence genes in Enterococcus spp. and characterization of isolates from broiler chickens. Appl. Environ. Microbiol. 76, 8033-8043.

EFSA (European Food Safety Authority) and ECDC (European Centre for Disease Prevention and Control) (2015): EU Summary Report on antimicrobial resistance in zoonotic and indicator bacteria from humans, animals and food in 2013. EFSA J. 13, 4036, 178 pp.

EMA (2014): European Medicines Agency, European Surveillance of Veterinary Antimicrobial Consumption. Sales of veterinary antimicrobial agents in 26 EU/EEA countries in 2012 (EMA/333921/2014).

Fertner, M. E., Olsen, R. H., Bisgaard, M. and Christensen, H. (2011): Transmission and genetic diversity of Enterococcus faecalis among layer chickens during hatch. Acta Vet. Scand. $53,56$.

Furtula, V., Jackson, C. R., Farrell, E. G., Barrett, J. B., Hiott, L. M. and Chambers, P. A. (2013): Antimicrobial resistance in Enterococcus spp. isolated from environmental samples in an area of intensive poultry production. Int. J. Environ. Res. Public Health 10, 1020-1036.

Kaukas, A., Hinton, M. and Linton, A. H. (1987): The effect of ampicillin and tylosin on the faecal enterococci of healthy young chickens. J. Appl. Bacteriol. 62, 441-447.

Kolbjørnsen, R., David, B. and Gilhuus, M. (2011): Bacterial osteomyelitis in a 3-week-old broiler chicken associated with Enterococcus hirae. Vet. Pathol. 48, 1134-1137.

Kosikowska, U., Stępień-Pyśniak, D., Ożga, D., Wernicki, A. and Malm, A. (2014): Identification of Bacillus spp. colonizing the nasal mucosa of healthy adults living in suburban area by using the matrix-assisted laser desorption-ionization time-of-flight mass spectrometry (MALDI-TOF MS) system. Curr. Issues Pharm. Med. Sci. 27, 137-141.

Kuhn, I., Iversen, A., Burman, L. G., Olsson-Liljequist, B., Franklin, A., Finn, M., Aarestrup, F., Seyfarth, A. M., Blanch, A. R., Vilanova, X., Taylor, H., Caplin, J., Moreno, M. A., Dominguez, L., Herrero, I. A. and Möllby, R. (2003): Comparison of enterococcal populations in animals, humans, and the environment - a European study. Int. J. Food Microbiol. 88, $133-145$.

Kuo, H. C., Chou, C. C., Chang, C. D., Gong, S. R., Wang, M. H. and Chang, S. K. (2009): Characterization of quinolone-resistant Enterococcus faecalis isolates from healthy chickens and pigs in Taiwan. J. Food Drug Anal. 17, 443-450.

Maasjost, J., Mühldorfer, K., Cortez de Jäckel, S. and Hafez, H. M. (2015): Antimicrobial susceptibility patterns of Enterococcus faecalis and Enterococcus faecium isolated from poultry flocks in Germany. Avian Dis. 59, 143-148.

Makrai, L., Nemes, C., Simon, A., Ivanics, É., Dudás, Z., Fodor, L. and Glávits, R. (2011): Association of Enterococcus cecorum with vertebral osteomyelitis and spondylolisthesis in broiler parent chicks. Acta Vet. Hung. 59, 11-21.

Marek, A., Pyzik, E., Stępień-Pyśniak, D., Hauschild, T. and Banach, T. (2015): Identification of strains with phenotypes similar to those of Staphylococcus aureus isolated from table chicken eggs using MALDI-TOF MS and genotyping methods. Bull. Vet. Inst. Pulawy 59, 235-239. 
Nowakiewicz, A., Ziółkowska, G., Zięba, P. and Kostruba, A. (2014): Undomesticated animals as a reservoir of multidrug-resistant Enterococcus in eastern Poland. J. Wildl. Dis. 50, 645-650.

Nowakiewicz, A., Ziółkowska, G., Zięba, P., Trościańczyk, A., Banach, T. and Kowalski, C. (2015): Modified 16S-23S rRNA intergenic region restriction endonuclease analysis for species identification of Enterococcus strains isolated from pigs, compared with identification using classical methods and matrix-assisted laser desorption/ionization time-of-flight mass spectrometry. J. Med. Microbiol. 64, 217-223.

Poeta, P., Costa, D., Rodrigues, J. and Torres, C. (2006): Antimicrobial resistance and the mechanisms implicated in faecal enterococci from healthy humans, poultry and pets in Portugal. Int. J. Antimicrob. Agents 27, 131-137.

Różańska, H., Lewtak-Piłat, A. and Osek, J. (2015): Antimicrobial resistance of Enterococcus faecalis isolated from meat. Bull. Vet. Inst. Pulawy 59, 229-233.

Ruzauskas, M., Siugzdiniene, R., Spakauskas, V., Povilonis, J., Seputiene, V., Suziedeliene, E., Daugelavicius, R. and Pavilonis, A. (2009): Susceptibility of bacteria of the Enterococcus genus isolated on Lithuanian poultry farms. Vet. Med. (Praha) 54, 583-588.

Sauer, S., Freiwald, A., Maier, T., Kube, M., Reinhardt, R., Kostrzewa, M. and Geider, K. (2008): Classification and identification of bacteria by mass spectrometry and computational analysis. PLoS One 3, e2843.

Seng, P., Drancourt, M., Gouriet, F., La Scola, B., Fournier, P. E., Rolain, J. M. and Raoult, D. (2009): Ongoing revolution in bacteriology: routine identification of bacteria by matrixassisted laser desorption ionization time-of-flight mass spectrometry. Clin. Infect. Dis. 49, 543-551.

Šeputienė, V., Bogdaite, A., Ruzauskas, M. and Suziedeliene, E. (2012): Antibiotic resistance genes and virulence factors in Enterococcus faecium and Enterococcus faecalis from diseased farm animals: pigs, cattle and poultry. Pol. J. Vet. Sci. 15, 431-438.

Stalker, M. J., Brash, M. L., Weisz, A., Ouckama, R. M. and Slavic, D. (2010): Arthritis and osteomyelitis associated with Enterococcus cecorum infection in broiler and broiler breeder chickens in Ontario, Canada. J. Vet. Diagn. Invest. 22, 643-645.

Steentjes, A., Veldman, K. T., Mevius, D. J. and Landman, W. J. (2002): Molecular epidemiology of unilateral amyloid arthropathy in broiler breeders associated with Enterococcus faecalis. Avian Pathol. 31, 31-39.

Sting, R., Richter, A., Popp, C. and Hafez, H. M. (2013): Occurrence of vancomycin-resistant enterococci in turkey flocks. Poult. Sci. 92, 346-351.

Szeleszczuk, P., Dolka, B., Żbikowski, A., Dolka, I. and Peryga, M. (2013): First case of enterococcal spondylitis in broiler chickens in Poland [in Polish]. Med. Weter. 9, 298-303.

Tankson, J. D., Thaxton, J. P. and Vizzier-Thaxton, Y. (2001): Pulmonary hypertension syndrome in broilers caused by Enterococcus faecalis. Infect. Immun. 69, 6318-6322.

Tankson, J. D., Thaxton, J. P. and Vizzier-Thaxton, Y. (2002): Bacteria in heart and lungs of young chicks. J. Appl. Microbiol. 92, 443-450.

Tejedor-Junco, M. T., Afonso-Rodríguez, O., Martín-Barrasa, J. L. and González-Martín, M. (2005): Antimicrobial susceptibility of Enterococcus strains isolated from poultry faeces. Res. Vet. Sci. 78, 33-38.

Torok, V. A., Allison, G. E., Percy, N. J., Ophel-Keller, K. and Hughes, R. J. (2011): Influence of antimicrobial feed additives on broiler commensal posthatch gut microbiota development and performance. Appl. Environ. Microbiol. 77, 3380-3390.

Torok, V. A., Hughes, R. J., Ophel-Keller, K., Ali, M. and Macalpine, R. (2009): Influence of different litter materials on cecal microbiota colonization in broiler chickens. Poult. Sci. 88, 2474-2481.

Tsakris, A., Woodford, N., Pournaras, S., Kaufmann, M. and Douboyas, J. (1998): Apparent increased prevalence of high-level aminoglycoside-resistant Enterococcus durans resulting from false identification by a semiautomated software system. J. Clin. Microbiol. 36, $1419-1421$. 
Usui, M., Ozawa, S., Onozato, H., Kuge, R., Obata, Y., Uemae, T., Ngoc, P. T., Heriyanto, A., Chalemchaikit, T., Makita, K., Muramatsu, Y. and Tamura, Y. (2014): Antimicrobial susceptibility of indicator bacteria isolated from chickens in Southeast Asian countries (Vietnam, Indonesia and Thailand). J. Vet. Med. Sci. 76, 685-692.

Velkers, F. C., van de Graaf-Bloois, L., Wagenaar, J. A., Westendorp, S. T., van Bergen, M. A., Dwars, R. M. and Landman, W. J. (2011): Enterococcus hirae-associated endocarditis outbreaks in broiler flocks: clinical and pathological characteristics and molecular epidemiology. Vet. Q. 31, 3-17.

Wieser, A., Schneider, L., Jung, J. and Schubert, S. (2012): MALDI-TOF MS in microbiological diagnostics-identification of microorganisms and beyond (mini review). Appl. Microbiol. Biotechnol. 93, 965-974.

Yoshimura, H., Ishimaru, M., Endoh, Y. S. and Kojima, A. (2000): Antimicrobial susceptibilities of enterococci isolated from faeces of broiler and layer chickens. Lett. Appl. Microbiol. 31, $427-432$. 